\title{
Semiclassical spectral correlator in quasi one-dimensional systems
}

\author{
Petr Braun ${ }^{1,2}$, Sebastian Müller ${ }^{3}$ and Fritz Haake ${ }^{1}$ \\ ${ }^{1}$ Fachbereich Physik, Universität Duisburg-Essen, 47048 Duisburg, Germany \\ ${ }^{2}$ Institute of Physics, Saint-Petersburg University, 198504 Saint-Petersburg, Russia \\ ${ }^{3}$ Department of Mathematics, University of Bristol, Bristol BS8 1TW, United \\ Kingdom \\ E-mail: Petr.Braun@uni-due.de
}

\begin{abstract}
.
We investigate the spectral statistics of chaotic quasi one dimensional systems such as long wires. To do so we represent the spectral correlation function $R(\epsilon)$ through derivatives of a generating function and semiclassically approximate the latter in terms of periodic orbits. In contrast to previous work we obtain both non-oscillatory and oscillatory contributions to the correlation function. Both types of contributions are evaluated to leading order in $1 / \epsilon$ for systems with and without time-reversal invariance. Our results agree with expressions from the theory of disordered systems.

PACS numbers: 05.45.Mt, 73.21.-b, 73.20.Fz
\end{abstract}

\section{Introduction}

In the field of quantum chaos and disorder, the behavior of quasi one-dimensional systems such as long wires is clearly distinguished from the behavior of "normal" chaotic or disordered systems. Most importantly, quasi one-dimensional systems display Anderson localization [1], i.e., wave functions are localized in only part of the wire and the conductance is suppressed. Anderson localization has important consequences for the statistics of energy levels [2, 3]. For normal systems the energy levels tend to repel each other; the spectral statistics is universal and agrees with predictions made by averaging over random-matrix caricatures of the possible Hamiltonians, according to the so called BGS conjecture [4. In contrast the spectral statistics of quasi one-dimensional systems depends on the length (and thus the diffusion time $T_{D}$ ); in the limit of large length the energy levels belonging to the localized wave functions become independent and hence show Poissonian statistics.

This difference between normal and quasi one-dimensional systems is well understood for disordered systems. Notable approaches are based on the DMPK equation [5] and on the nonlinear sigma model, a field-theoretical technique to evaluate averages over different realizations of the disorder potential. From the latter, localization 
could be extracted in [6, 7]. The appropriate definition of quasi one-dimensional behavior arising in this context is that the classical diffusion time $T_{D}$ becomes comparable to or larger than the relevant quantum time scales, in particular the Heisenberg time $T_{H}=\frac{2 \pi \hbar}{\Delta}$ where $\Delta$ is the mean level spacing. A random-matrix model for systems of this type was considered in [8, 9, 10].

For clean chaotic systems (e.g. wires in which the classical motion becomes chaotic due to the shape of the boundary) the effects of quasi one-dimensionality are less well understood, and most of the literature is restricted to normal systems. A quantity that has attracted a lot of attention in this context is the spectral correlation function $R(\epsilon)$. For dynamical systems with a level density $\rho(E)$ this correlation function is defined by

$$
R(\epsilon)=\Delta^{2}\left\langle\rho\left(E+\frac{\epsilon \Delta}{2 \pi}\right) \rho\left(E-\frac{\epsilon \Delta}{2 \pi}\right)\right\rangle-1
$$

where $\epsilon$ is a real energy offset, the brackets denote an average over the center energy $E$ and $\Delta$ is the mean level spacing. For simplicity we assume that $\Delta$ is brought to 1 by appropriately scaling the energy levels. Random matrix theory (RMT) now makes predictions for $R(\epsilon)$ : For systems without time reversal invariance an average over the Gaussian Unitary Ensemble (GUE) of RMT gives $-\frac{1}{2 \epsilon^{2}}+\frac{\cos 2 \epsilon}{2 \epsilon^{2}}$ while for time reversal invariant systems an average over the Gaussian Orthogonal Ensemble (GOE) leads to infinite power series in $\frac{1}{\epsilon^{n}}$ and $\frac{\cos 2 \epsilon}{\epsilon^{n}}$. The slow (power law) decay of oscillations leads to a singularity in the Fourier transform of $R(\epsilon)$ (the spectral form factor) at time $t=T_{H}$.

To show that individual systems are faithful to these predictions a semiclassical approach was proposed in [11, 12, 13]. The essential idea is to express $\rho(E)$ as a sum over periodic orbits, using Gutzwiller's formula [14], and then study the interference between contributions of these orbits. The leading non-oscillatory contribution arises from "diagonal" pairs of identical (up to time reversal) orbits [11]. The remaining terms were accessed only recently in [13, 15, 16, 17].

In the present paper we want to generalize these new results to quasi onedimensional systems. For these systems the diagonal approximation to the small-time form factor (the Fourier image of the non-oscillatory part of $R(\epsilon)$ ) was evaluated by Dittrich [18. First results on off-diagonal contributions were obtained by Schanz and Smilansky [19] for one-dimensional quantum graphs, and by Brouwer and Altland [20] who semiclassically explained localization for quasi one-dimensional systems modelled by an array of quantum dots. In contrast, we will focus on general quasi one-dimensional systems such as long wires. We use a periodic-orbit expansion not of the correlation function itself, but of a generating function which yields $R(\epsilon)$ upon taking derivatives [16, 17]. This enables us to determine, to leading order in $\frac{1}{\epsilon}$, both the non-oscillatory and the oscillatory parts of $R(\epsilon)$. In this order we see that the effects of quasi onedimensionality reduce to modification of the periodic orbit sum rule suggested in [18. For systems without time reversal invariance it suffices to perform a diagonal approximation on the level of the generating function. In contrast, for time-reversal invariant systems this diagonal approximation still captures only the non-oscillatory part; the evaluation of the oscillatory part involves off-diagonal contributions of pairs 
of non-identical but similar orbits. Both for systems with and without time-reversal invariance we reach agreement with results for disordered systems by Andreev and Altshuler [3]. Our results illustrate how semiclassical methods are useful not only for describing universal features of "normal" systems but also deviations from universality.

Higher-order corrections in $1 / \epsilon$ should be similarly accessible; their calculation needs taking into account more complicated groups of correlated orbits introduced in previous work on normal systems [13, 15], combined with treatment of higher order effects of quasi one-dimensionality. An extension of this approach to Anderson localization appears within reach.

\section{Two-point spectral correlator and the generating function}

To get started we briefly review how the correlation function $R(\epsilon)$ can be accessed through a generating function. Following Ref. [16] we write $R(\epsilon)$ as the real part of the complex correlation function

$$
\begin{aligned}
C\left(\epsilon^{+}\right) & =\frac{\Delta^{2}}{2 \pi^{2}}\left\langle\operatorname{Tr}\left(E+\frac{\epsilon^{+} \Delta}{2 \pi}-\hat{H}\right)^{-1} \operatorname{Tr}\left(E-\frac{\epsilon^{+} \Delta}{2 \pi}-\hat{H}\right)^{-1}\right\rangle-\frac{1}{2}, \\
\epsilon^{ \pm} & =\epsilon \pm \mathrm{i} \gamma \\
R(\epsilon) & =\lim _{\gamma \rightarrow 0} \operatorname{Re} C\left(\epsilon^{+}\right)
\end{aligned}
$$

and determine the latter from a generating function, the energy-averaged combination of four spectral determinants

$$
Z\left(\epsilon_{A}^{+}, \epsilon_{B}^{-}, \epsilon_{C}^{+}, \epsilon_{D}^{-}\right)=\left\langle\frac{\operatorname{det}\left(E+\epsilon_{C}^{+}-\hat{H}\right) \operatorname{det}\left(E+\epsilon_{D}^{-}-\hat{H}\right)}{\operatorname{det}\left(E+\epsilon_{A}^{+}-\hat{H}\right) \operatorname{det}\left(E+\epsilon_{B}^{-}-\hat{H}\right)}\right\rangle
$$

as

$$
R(\epsilon)=\lim _{\gamma \rightarrow 0} \operatorname{Re} C\left(\varepsilon^{+}\right)=-\frac{1}{2}+\left.2 \operatorname{Re} \lim _{\gamma \rightarrow 0} \frac{\partial^{2} Z}{\partial \epsilon_{A}^{+} \partial \epsilon_{B}^{-}}\right|_{\|, \times} .
$$

Here the subscripts \pm indicate small positive or negative imaginary parts. The symbols $\|, \times$ denote two alternative ways of identifying the energy arguments, to be referred to as "columnwise" $(\|)$ and "crosswise" $(\times)$,

$$
\begin{aligned}
& \|: \epsilon_{A}^{+}=\epsilon_{C}^{+}=\epsilon_{A}^{+}, \epsilon_{B}^{-}=\epsilon_{D}^{-}=-\epsilon^{+} \quad \text { columnwise, } \\
& \times: \epsilon_{A}^{+}=\epsilon^{+}, \epsilon_{B}^{-}=-\epsilon^{+}, \epsilon_{C}^{+}=-\epsilon^{-}, \epsilon_{D}^{-}=\epsilon^{-}, \gamma \rightarrow+0 \quad \text { crosswise . }
\end{aligned}
$$

Both procedures would yield the same result for the two-point correlator if implemented rigorously. However, we shall have to calculate $Z$ semiclassically, and that approximation entails two different expressions, one $(\|)$ reproducing the non-oscillatory part and the other $(\times)$ the oscillatory part of $R(\epsilon)$. To obtain the full result both expressions have to be added. In [17] it was shown that this addition can be understood naturally in terms of an improved semiclassical approximation preserving the unitarity of the time evolution (the Riemann-Siegel lookalike formula [21]). 
Semiclassical spectral correlator in quasi one-dimensional systems

The semiclassical approximation for $Z$ is based on Gutzwiller's formula for the trace of the resolvent

$$
\operatorname{tr}\left(E^{+}-H\right)^{-1}=-\frac{i \pi E^{+}}{\Delta}+\sum_{a} F_{a} e^{i S_{a}\left(E^{+}\right) / \hbar}
$$

The factor proportional to $E^{+}$is the smooth (Weyl) part of the level density and the sum taken over periodic orbits with $S_{a}, T_{a}, F_{a}$ action, period and stability coefficient of the ath orbit. Integration then yields the semiclassical approximation of the determinant

$$
\begin{aligned}
\operatorname{det}\left(E^{+}-\hat{H}\right)^{-1} & =\exp \left[-\int^{E^{+}} d E \operatorname{Tr}(E-H)^{-1}\right] \\
& \sim \exp \left(\frac{\mathrm{i} \pi E^{+}}{\Delta}+\sum_{a} F_{a} \mathrm{e}^{\frac{i}{\hbar} S_{a}\left(E^{+}\right)}\right)
\end{aligned}
$$

Substituting such expansions for all four determinants in $Z$ and expanding, e.g., $S_{a}\left(E+\epsilon_{A}^{+}\right) \approx S_{a}(E)+T_{a} \epsilon_{A}^{+}$we obtain

$$
\begin{aligned}
Z \approx \mathrm{e}^{\frac{i}{2}\left(\epsilon_{A}^{+}-\epsilon_{B}^{-}-\epsilon_{C}^{+}+\epsilon_{D}^{-}\right)} \exp \left[\sum_{a} F_{a} \mathrm{e}^{\frac{\mathrm{i}}{\hbar} S_{a}(E)}\left(\mathrm{e}^{\mathrm{i} \frac{T_{a}}{T_{H}} \epsilon_{A}^{+}}-\mathrm{e}^{\mathrm{i} \frac{T_{a}}{T_{H}} \epsilon_{C}^{+}}\right)\right. \\
\left.+\sum_{a} F_{a}^{*} \mathrm{e}^{-\frac{i}{\hbar} S_{a}(E)}\left(\mathrm{e}^{-\mathrm{i} \frac{T_{a}}{T_{H}} \epsilon_{B}^{-}}-\mathrm{e}^{-\mathrm{i} \frac{T_{a}}{T_{H}} \epsilon_{D}^{-}}\right)\right] .
\end{aligned}
$$

\section{Systems without time reversal invariance}

\subsection{Diagonal approximation}

The semiclassical representation (8) falls into a product over periodic orbits,

$$
\begin{aligned}
Z=e^{\frac{i}{2}\left(\varepsilon_{A}^{+}-\varepsilon_{B}^{-}-\varepsilon_{C}^{+}+\varepsilon_{D}^{-}\right)} Z_{0}, \quad Z_{0}=\prod_{a} z_{a}, \\
z_{a}=\exp [\underbrace{F_{a}\left(\mathrm{e}^{\mathrm{i} \frac{T_{a}}{T_{H}} \epsilon_{A}^{+}}-\mathrm{e}^{\mathrm{i} \frac{T_{a}}{T_{H}} \epsilon_{C}^{+}}\right)}_{=f_{A C}^{a}} \mathrm{e}^{\frac{i}{\hbar} S_{a}(E)} \\
+\underbrace{F_{a}^{*}\left(\mathrm{e}^{-\mathrm{i} \frac{T_{a}}{T_{H}} \epsilon_{B}^{-}}-\mathrm{e}^{-\mathrm{i} \frac{T_{a}}{T_{H}} \epsilon_{D}^{-}}\right)}_{=f_{B D}^{a *}} \mathrm{e}^{-\frac{i}{\hbar} S_{a}(E)}] .
\end{aligned}
$$

The diagonal approximation [11] assumes that for systems without time-reversal

invariance contributions of different periodic orbits are uncorrelated. For the generating function this means that the energy-averaged $Z_{0}$ becomes a product of single-orbit averages,

$$
\left\langle Z_{0}\right\rangle_{\mathrm{diag}}=\prod_{a}\left\langle z_{a}\right\rangle .
$$

The energy average in $\left\langle z_{a}\right\rangle$ does away with rapid oscillations due to the phase factors $\mathrm{e}^{ \pm \mathrm{i} S(E) / \hbar}$ in the exponent. Since $z_{a}$ is periodic in the phase $\Phi_{a}=S_{a}(E) / \hbar$ we may average with respect to $\Phi_{a}$, over a single period $2 \pi$. This yields

$$
\left\langle z_{a}\right\rangle=\frac{1}{2 \pi} \int_{0}^{2 \pi} d \Phi \exp \left(f_{A C}^{a} \mathrm{e}^{\mathrm{i} \Phi}+f_{B D}^{a *} \mathrm{e}^{-\mathrm{i} \Phi}\right)=I_{0}\left(2 \sqrt{f_{A C}^{a} f_{B D}^{a *}}\right),
$$


where $I_{0}$ is the imaginary-argument Bessel function. The expansion $\ln I_{0}(y)=\frac{y^{2}}{4}-$ $\frac{y^{4}}{64}+\ldots$ gives $\left\langle Z_{0}\right\rangle=\exp \left[\sum_{a} f_{A C}^{a} f_{B D}^{a *}-\frac{1}{4} \sum_{a}\left(f_{A C}^{a} f_{B D}^{a *}\right)^{2}+\ldots\right]$. In the semiclassical limit $\left(T_{H} \rightarrow \infty\right)$ it suffices to keep only the leading quadratic term in the exponent $\ddagger$

Our task is thus reduced to calculating the periodic-orbit sum

$$
\begin{gathered}
\ln \left\langle Z_{0}\right\rangle_{\operatorname{diag}}=\sum_{a} f_{A C}^{a} f_{B D}^{a *} \\
=\sum_{a}\left|F_{a}\right|^{2}\left(\mathrm{e}^{\mathrm{i} \frac{T_{a}}{T_{H}} \epsilon_{A}^{+}}-\mathrm{e}^{\mathrm{i} \frac{T_{a}}{T_{H}} \varepsilon_{C}^{+}}\right)\left(\mathrm{e}^{-\mathrm{i} \frac{T_{a}}{T_{H}} \epsilon_{B}^{-}}-\mathrm{e}^{-\mathrm{i} \frac{T_{a}}{T_{H}} \epsilon_{D}^{-}}\right)
\end{gathered}
$$

\subsection{Diffusive vs ergodic behavior}

For "normal" systems the sum over periodic orbits can be done using the well-known sum rule of Hannay and Ozorio de Almeida [22]

$$
\sum_{a}\left|F_{a}\right|^{2}(\cdot)=\int_{T_{0}}^{\infty} \frac{d T}{T}(\cdot)
$$

which expresses the approximately ergodic behavior of long orbits; short orbits below a certain classical period $T_{0}$ have to be excluded.

The quasi one-dimensional character of long wires requires a modification of that sum rule [18]. The momentum components in all directions and the transverse coordinates can still effectively randomize after a few bounces against the boundary, but the longitudinal coordinate takes much longer to explore the whole length $L$ of the wire. Given chaos inducing boundaries, each bounce will with finite probability change the sign of the longitudinal momentum component and thus entail diffusion along the wire. The longitudinal coordinate becomes randomized only for times long enough to explore the wire, i.e., times in excess of the diffusion (Thouless) time $T_{D}=L^{2} / D$ where $D$ denotes the diffusion constant. Orbits with periods $T$ much smaller than that Thouless time will have explored only the small fraction $\sqrt{D T} / L$ of the whole length, and the inverse of that fraction must be expected as a factor of increase of the right hand side of the above sum rule, relative to orbits with periods $T \gg T_{D}$.

Dittrich [18] has determined the aforementioned factor of increase for arbitrary values of the ratio $T / T_{D}$ as the integral $\int_{0}^{L} d x_{0} p_{x_{0}}\left(x_{0}, T \mid x_{0}\right)=P(T)$ of the probability density of return to an arbitrary point $x_{0}$ of departure after a time $T$, for a onedimensional random walk. Solution of the diffusion equation for a wire of length $L$, led to the "enhanced-return" factor

$$
P(T)=\sum_{n=0}^{\infty} \exp \left(-\frac{\pi^{2} n^{2} T}{2 T_{D}}\right)=\frac{1}{2}\left[1+\vartheta_{3}\left(0, \mathrm{e}^{-\pi^{2} T / 2 T_{D}}\right)\right]
$$

$\ddagger$ For this term the decrease of the stability coefficients $\left|F_{a}\right|^{2}$ with the period is just compensated by the exponential increase in the number of orbits. For all other terms $F_{a}$ decreases faster; the contributions of the long orbits then become exponentially small whereas the shortest orbits make for corrections of the order $\mathrm{o}\left(T_{0} / T_{H}\right)$. 
where $\vartheta_{3}(u, q)$ denotes the elliptic theta-function of the third kind [23]. The sum rule modified for quasi one dimensional systems thus reads

$$
\sum_{a}\left|F_{a}\right|^{2}(\cdot)=\int_{T_{0}}^{\infty} \frac{d T}{T} P(T)(\cdot) .
$$

Hannay's and Ozorio de Almeida's sum rule is restored if $T_{D}$ is so small compared with $T$ that only the $n=0$ term in (14) survives and $P(T) \sim 1$. In the opposite limit $T_{D} \gg T$ we have $P(T) \sim \sqrt{T_{D} / 2 \pi T}=L / \sqrt{2 \pi D T}$ which agrees with the above qualitative expectation.

Inasmuch as orbit periods of the order of the Heisenberg time $T_{H}$ determine the level statistics the time scale ratio

$$
\zeta=\frac{\pi^{2} T_{H}}{2 T_{D}}
$$

will play an important role for the wires under study here. In particular, the crossover from normal behavior $(\zeta \gg 1)$ to the quasi one-dimensional behavior under study here takes place for $\zeta$ of the order unity; for $\zeta \ll 1$ the spectral statistics must be compatible with localization.

We now invoke the modified sum rule and the identity $\int_{0}^{\infty} \frac{d T}{T}\left(\mathrm{e}^{\mathrm{i} a T}-\mathrm{e}^{\mathrm{i} b T}\right)=\ln \frac{a}{b}$; the lower limit $T_{0}$ of the time integral could be replaced by zero, accepting a negligible error $\mathrm{o}\left(T_{0} / T_{H}\right)$. We thus get the diagonal approximation for our generating function as

$$
\begin{array}{r}
\ln \langle Z\rangle_{\operatorname{diag}} \approx \sum_{n=0}^{\infty} \int_{0}^{\infty} \frac{d T}{T} \mathrm{e}^{-n^{2} \frac{\zeta T}{T_{H}}}\left[\mathrm{e}^{\mathrm{i} \frac{T}{T_{H}}\left(\epsilon_{A}^{+}-\epsilon_{B}^{-}\right)}-\mathrm{e}^{\mathrm{i} \frac{T}{T_{H}}\left(\epsilon_{C}^{+}-\epsilon_{B}^{-}\right)}\right. \\
\left.+\mathrm{e}^{-\mathrm{i} \frac{T}{T_{H}}\left(\epsilon_{D}^{-}-\epsilon_{C}^{+}\right)}-\mathrm{e}^{-\mathrm{i} \frac{T}{T_{H}}\left(\epsilon_{D}^{-}-\epsilon_{A}^{+}\right)}\right] \\
=\sum_{n=0}^{\infty} \ln \frac{\left(\mathrm{i} \zeta n^{2}+\epsilon_{C}^{+}-\epsilon_{B}^{-}\right)\left(\mathrm{i} \zeta n^{2}+\epsilon_{A}^{+}-\varepsilon_{D}^{-}\right)}{\left(\mathrm{i} \zeta n^{2}+\epsilon_{A}^{+}-\epsilon_{B}^{-}\right)\left(\mathrm{i} \zeta n^{2}+\epsilon_{C}^{+}-\epsilon_{D}^{-}\right)} .
\end{array}
$$

The infinite product implicitly involved here can be brought to a closed form using

$$
\prod_{n=0}^{\infty} \frac{n^{2}+a}{n^{2}+b}=\frac{\varphi(a)}{\varphi(b)}, \quad \varphi(x)=\sqrt{x} \sinh \pi \sqrt{x},
$$

whereupon we arrive at our final result for the generating function in the diagonal approximation,

$$
\langle Z\rangle_{\operatorname{diag}}=\mathrm{e}^{\frac{\mathrm{i}}{2}\left(\epsilon_{A}^{+}-\epsilon_{B}^{-}-\epsilon_{C}^{+}+\epsilon_{D}^{-}\right)} \frac{\varphi\left(\frac{\epsilon_{C}^{+}-\epsilon_{B}^{-}}{\mathrm{i} \zeta}\right) \varphi\left(\frac{\epsilon_{A}^{+}-\epsilon_{D}^{-}}{\mathrm{i} \zeta}\right)}{\varphi\left(\frac{\epsilon_{A}^{+}-\epsilon_{B}^{-}}{\mathrm{i} \zeta}\right) \varphi\left(\frac{\epsilon_{C}^{+}-\epsilon_{D}^{-}}{\mathrm{i} \zeta}\right)} .
$$

In the limit $T_{D} \rightarrow 0$ or $\zeta \rightarrow \infty$ we have $\varphi(x) \rightarrow \pi x$, and the generating function tends to its familiar form for normal systems [15].

\subsection{Two-point correlator and form factor}

Substituting $\langle Z\rangle_{\operatorname{diag}}$ for $\langle Z\rangle$ in (4) and identifying energies columnwise (\|) we obtain

$$
C_{\|}(\epsilon)=-2 \sum_{n=0}^{\infty} \frac{1}{\left(\mathrm{i} \zeta n^{2}+2 \epsilon\right)^{2}}
$$


Semiclassical spectral correlator in quasi one-dimensional systems

$$
\begin{aligned}
& =-\frac{1}{2 \epsilon^{2}}\left(\frac{1}{2}+\frac{1}{4} \theta \cot \theta+\frac{\mathrm{i} \epsilon \pi^{2}}{2 \zeta \sin ^{2} \theta}\right), \\
\theta \quad & =(1+\mathrm{i}) \pi \sqrt{\frac{\epsilon}{\zeta}} .
\end{aligned}
$$

Upon taking the real part we are led to a cumbersome expression for $R(\epsilon)$ equivalent to the earlier RMT [3] and semiclassical [18] results for the non-oscillatory part of the correlator. In the limit $\zeta \rightarrow \infty$ the GUE behavior $R_{\text {non-osc }}(\epsilon)=-1 / 2 \epsilon^{2}$ is restored.

The crosswise $(\times)$ identification of parameters, on the other hand, entails

$$
C_{\times}(\epsilon)=\frac{2 \pi^{2} \mathrm{e}^{\mathrm{i} 2 \epsilon}}{\epsilon \zeta\left[\cosh 2 \pi \sqrt{\frac{\epsilon}{\zeta}}-\cos 2 \pi \sqrt{\frac{\epsilon}{\zeta}}\right]},
$$

and now the real part yields the oscillatory part of the correlator $R_{\mathrm{osc}}(\epsilon)$, in agreement with what Andreev and Altshuler [3] had found through an average over an ensemble of disordered systems. The GUE expression $R_{\mathrm{osc}}(\epsilon)=\cos 2 \epsilon / 2 \epsilon^{2}$ follows in the limit $\zeta \rightarrow \infty$.

For finite $\zeta$ the amplitude of oscillations of the spectral correlation function tends to zero exponentially with $\epsilon \rightarrow \infty$ instead of the power law characteristic of normal systems. That leads to qualitative changes in the spectral form factor $K(\tau)$ where $\tau$ is the dimensionless time, $\tau=t / T_{H}$. For $\tau>0$, the form factor can be defined as the Fourier transform,

$$
K(\tau)=\frac{1}{2 \pi} \int_{-\infty+i \gamma}^{\infty+i \gamma} e^{-i 2 \varepsilon \tau} C(\varepsilon) d \varepsilon .
$$

In normal systems without time reversal invariance $K(\tau)$ experiences discontinuity of its first derivative at $\tau=1$ introduced by the Fourier transform of the oscillatory part of the spectral correlation function. In quasi one-dimensional system this discontinuity is replaced by a smooth transition from the small-time to large-time behavior. Another change associated with the non-oscillatory part of the spectral correlation function is the much faster growth of $K(\tau)$ at small $\tau$ : The respective ("parallel") part of the diagonal form factor first deduced semiclassically in [18],

$$
K_{\|}(\tau)=\tau \sum_{n=0}^{\infty} e^{-\zeta n^{2} \tau}=\tau P\left(T_{H} \tau\right)
$$

grows like square root rather than linearly. That faster rise toward the saturation value unity may be seen as a (slightly indirect) hint to localization; in the Poissonian limit this rise would be a jump, $K(\tau)=1$ for all $\tau>0$.

As a note of caution it has to be mentioned that, in contrast to normal systems without time reversal, the diagonal approximation for the correlation functions no longer coincides with the exact result in quasi one-dimensional systems. In particular, the Fourier transform of the oscillatory part (20) is no longer zero for $\tau<1$ tending to a finite negative value for $\tau \rightarrow+0$. Consequently the total form factor of the diagonal approximation becomes negative for small $\tau$ although the exact form factor is known to be non-negative. 


\section{Time reversal invariance}

Interesting changes arise if time-reversal invariance holds. Periodic orbits then exist in time reversed pairs $(a, \bar{a})$ with exactly the same action and stability coefficients. The generating function becomes a product of contributions of different pairs, and these pairs are uncorrelated in the diagonal approximation. Due to $z_{a}=z_{\bar{a}}$ the generating function is squared compared to (18), the Weyl factor apart,

$$
\langle Z\rangle_{\operatorname{diag}}=\mathrm{e}^{\frac{i}{2}\left(\epsilon_{A}^{+}-\epsilon_{B}^{-}-\epsilon_{C}^{+}+\epsilon_{D}^{-}\right)}\left[\frac{\varphi\left(\frac{\epsilon_{C}^{+}-\epsilon_{B}^{-}}{i \zeta}\right) \varphi\left(\frac{\epsilon_{A}^{+}-\epsilon_{D}^{-}}{i \zeta}\right)}{\varphi\left(\frac{\epsilon_{A}^{+}-\epsilon_{B}^{-}}{i \zeta}\right) \varphi\left(\frac{\epsilon_{C}^{+}-\epsilon_{D}^{-}}{i \zeta}\right)}\right]^{2} .
$$

Using this generating function with columnwise identification of arguments we find that the non-oscillatory part of the two-point correlation function and the small-time form factor are doubled compared to (19) and (22); this is in line with Refs. [3] and [18].

Remarkably, for time-reversal invariant systems the diagonal approximation yields no oscillatory contributions to the correlation function, i.e., there are no terms of order $\frac{\cos 2 \epsilon}{\epsilon^{2}}$. This can be understood as follows. In the crosswise limit ([6]) we have

$$
\epsilon_{C}^{+}-\epsilon_{B}^{-}=\epsilon_{A}^{+}-\epsilon_{D}^{-}=\mathrm{i} 2 \gamma, \quad \gamma \rightarrow 0
$$

such that we can replace $\varphi(x) \rightarrow \pi x$ in the numerator of $\langle Z\rangle_{\text {diag }}$; this gives

$$
\langle Z\rangle_{\operatorname{diag}} \propto\left(\epsilon_{C}^{+}-\epsilon_{B}^{-}\right)^{2}\left(\epsilon_{A}^{+}-\epsilon_{D}^{-}\right)^{2}
$$

such that $\langle Z\rangle_{\text {diag }}$ tends to zero like $\mathrm{O}\left(\gamma^{4}\right)$. The two derivatives w.r.t. $\epsilon_{A}^{+}, \epsilon_{B}^{-}$can only eliminate two factors $\gamma$. This leaves a result that tends to zero like $\mathrm{O}\left(\gamma^{2}\right)$ which means that $C_{\text {diag, } \times}=0$.

To derive the oscillatory component of the spectral correlator we thus have to go beyond the diagonal approximation and take into account correlations between the factors $z_{a}$ in the generating function related to different periodic orbits. For the relevant correlated orbits the differences $\left\langle z_{a} z_{b}\right\rangle-\left\langle z_{a}\right\rangle\left\langle z_{b}\right\rangle,\left\langle z_{a} z_{b} z_{c}\right\rangle-\left\langle z_{a}\right\rangle\left\langle z_{b}\right\rangle\left\langle z_{c}\right\rangle$ etc. must be non-zero. In view of the semiclassical limit this is possible only if the respective actions have a chance to cancel, e.g. if $S_{a}(E) \approx S_{b}(E)$ or $S_{a} \approx S_{b}(E)+S_{c}(E)$. Such correlations between orbits indeed exist for chaotic dynamics; they stem from "encounters", i.e., places where two or more stretches of the same orbit or of different orbits are close and almost parallel or antiparallel to each other. By changing the connections inside these encounters one can turn, e.g., an orbit $a$ into an orbit $b$ with almost the same action, or split it into two orbits $b$ and $c$ whose sum of actions is close to the action of $a$. We shall refer to such sets of correlated orbits as "bunches". The simplest encounter involves two almost antiparallel orbit stretches; the bunch it generates is the famous Sieber-Richter pair (containing one orbit where the encounter forms a crossing in configuration space and one where it forms an avoided crossing) [13]. More complicated scenarios were introduced in [15, 16]. It has been shown in [16] that taking into account both the "diagonal" correlations and those related to bunches gives 
correct semiclassical asymptotics of the generating function and the correlation function of normal systems. The generating function was found as

$$
\langle Z\rangle=\langle Z\rangle_{\operatorname{diag}}\left(1+\langle Z\rangle_{\text {off }}\right),
$$

where the off-diagonal part $\langle Z\rangle_{\text {off }}$ contains the contributions of the bunches mentioned.

Let us now determine the term in $\langle Z\rangle_{\text {off }}$ responsible for the leading oscillatory contribution to the correlator. Multiplication with this term must remove one factor $\epsilon_{C}^{+}-\epsilon_{B}^{-} \rightarrow i 2 \gamma$ and one factor $\epsilon_{A}^{+}-\epsilon_{D}^{-} \rightarrow i 2 \gamma$ from (25). The product is then proportional to $\left(\epsilon_{A}^{+}-\epsilon_{D}^{-}\right)\left(\epsilon_{C}^{+}-\epsilon_{B}^{-}\right)$and survives differentiation w.r.t. $\epsilon_{A}^{+}$and $\epsilon_{B}^{-}$and taking the limit $\gamma \rightarrow 0$. For normal systems the term of lowest order in $\frac{1}{\epsilon}$ satisfying this condition reads (see Eqs. $(13,14)$ of the on-line version of Ref. [16])

$$
\langle Z\rangle_{\mathrm{off}, \times}=-\frac{4}{\left(\epsilon_{A}^{+}-\epsilon_{D}^{-}\right)\left(\epsilon_{C}^{+}-\epsilon_{B}^{-}\right)} .
$$

In a quasi one-dimensional system, due to the diffusive dynamics, Eq. (27) would be replaced by an expression analogous to the diagonal approximation, i.e. a summand as in (27), plus terms where the energy differences in the denominator are shifted by finite imaginary amounts of the type $\mathrm{i} \zeta n^{2}$, with integer $n$ as in (17). However, the shifted terms would, for $n \neq 0$, no longer diverge in the limit $(\times)$. Hence combined with the above factor $\mathrm{O}\left(\gamma^{2}\right)$ they would yield vanishing contributions to the correlation function. Therefore the leading term in $C_{\times}(\epsilon)$ is still due to (27). Substituting (27) into (26) and calculating derivatives in the crosswise procedure, we obtain the oscillatory component of the complex correlator,

$$
\lim _{\gamma \rightarrow+0} C_{\times}(\epsilon)=\frac{8 \pi^{4} \mathrm{e}^{\mathrm{i} 2 \epsilon}}{\epsilon^{2} \zeta^{2}\left(\cosh 2 \pi \sqrt{\frac{\epsilon}{\zeta}}-\cos 2 \pi \sqrt{\frac{\epsilon}{\zeta}}\right)^{2}} .
$$

Its real part coincides with the RMT result for the two-point spectral correlation function in the presence of time reversal[3], now deduced semiclassically for individual chaotic quasi one-dimensional systems. In the normal-system limit $\zeta \rightarrow \infty(28)$ tends to the random-matrix expression $\exp (\mathrm{i} 2 \epsilon) / 2 \epsilon^{4}$. On the other hand, for all finite $\zeta$ the amplitude of oscillations diminishes exponentially with the growth of $\epsilon$. As a consequence the discontinuity at $\tau=1$ of the third derivative of the GOE spectral form factor $K(\tau)$ [9] is smoothed out in the quasi one-dimensional case.

\section{Acknowledgments}

Financial support of the Sonderforschungsbereich SFB/TR12 of the Deutsche Forschungsgemeinschaft is gratefully acknowledged.

\section{References}

[1] Anderson P W 1958 Phys. Rev. 1091492

[2] Altshuler B L and Shklovski B I 1986 Sov. Phys. -JETP 64127 
[3] Andreev A V and Altshuler B L 1995 Phys. Rev. Lett. 75902

[4] Bohigas O, Giannoni M J and Schmit C 1984 Phys. Rev. Lett. 52 1; McDonald S W and Kauffmann A N 1979 Phys. Rev. Lett. 42 1189; Casati G, Valz-Gris F and Guarneri I 1980 Lett. Nuovo Cim. 28 279; Berry M V 1987 Proc. R. Soc. Lond. A 413183

[5] Dorokhov O N 1982 JETP Lett. 36 318; Mello P A, Pereyra P and Kumar N 1988 Ann. Phys., NY 181290

[6] Lamacraft A, Simons B D and Zirnbauer M R 2004 Phys. Rev. 70075412

[7] Altland A, Kamenev A and Tian C 2005 Phys. Rev. Lett. 95206601

[8] Fyodorov Y V and Mirlin A D 1994 Int. J. Mod. Phys. B8 3795

[9] Haake F 2001 Quantum Signatures of Chaos (Springer, Berlin, 2nd ed.)

[10] Efetov K B 1997 Supersymmetry in Disorder and Chaos (Cambridge University Press, Cambridge)

[11] Berry M V 1985 Proc. R. Soc. A 400229

[12] N. Argaman et al. 1993 Phys. Rev. Lett. 714326

[13] Sieber M and Richter K 2001 Phys. Scr. T 90128

[14] Gutzwiller M 1990 Chaos in Classical and Quantum Mechanics (Springer, New York)

[15] Müller S, Heusler S, Braun P, Haake F and Altland A 2004 Phys. Rev. Lett. 93 014103; Müller S, Heusler S, Braun P, Haake F and Altland A 2005 Phys. Rev. E 72046207

[16] Heusler S, Müller S, Altland A, Braun P and Haake F 2007 Phys. Rev. Lett. 98 044103; Heusler S, Müller S, Altland A, Braun P and Haake F 2007 arXiv:nlin. CD/0610053.

[17] Keating J P and Müller S 2007 Proc. R. Soc. A 4633241

[18] Dittrich T 1996 Physics Reports 271267

[19] Schanz H and Smilansky U 2000 Phys. Rev. Lett. 841427

[20] Brouwer P and Altland A, 2007 arXiv:0802.0976v1

[21] Berry M V 1986 Riemann's zeta function: a model for quantum chaos? In Quantum chaos and statistical nuclear physics (eds Seligman T H \& Nishioka H, Springer Lecture Notes in Physics, Berlin, Germany: Springer) no. 263, pp. 1-17;

Berry M V and Keating J P 1990 J. Phys. A 23 4839;

Keating J P 1992 Proc. R. Soc. Lond. A 436 99;

Berry M V and Keating J P 1992 Proc. R. Soc. Lond. A 437151

[22] Hannay J H and Ozorio de Almeida A M 1984 J. Phys. A 173429

[23] Abramovitz M and Stegun I A 1972 Handbook of mathematical functions (Dover, New York) 